\title{
Kartenwelten: Von Kontinenten und Archipelen
}

Was bedeutet dies nun, wenn wir die von Auerbach entlang der abendländischen Literatur durchexerzierte Zweiteilung auf die Welt der Karten, auf die kartographischen Weltentwürfe umsetzen und entsprechend übersetzen? Man könnte hier im Grunde von zwei unterschiedlichen Positionen sprechen, die wir etwas vereinfachend als die kontinentale (also die Kontinente und das Kontinuierliche betonende) und die archipelische (also die vielinseligen Differenzen betonende) Position bezeichnen könnten.

Zunächst darf ich dabei auf die Karte von Martin Waldseemüller alias Hylacomylus eingehen, ${ }^{1}$ eine Karte, die ungeheuer stark an den Kontinenten ausgerichtet ist und die vier Erdteile, die „vier Teile der Welt“, in den Vordergrund rückt. Sie schafft ein europäisches Bewusstsein von jener Neuen Welt, die Columbus selbst noch nicht ins Bewusstsein getreten war, wohl aber Amerigo Vespucci, der von einem „mundus novus“ sprach. Die Ausrichtung an Kontinenten wird vielleicht am besten deutlich, wenn wir diese kontinentale Weltsicht des Martin Waldseemüller in einen kontrastiven Bezug zu einer Inselkarte bringen, die uns vor Augen führt, was es heißt, am Nicht-Kontinuierlichen, am Inselhaften und Archipelischen ausgerichtet zu sein.

Doch bleiben wir noch einen Augenblick bei Martin Waldseemüller, dessen Name mit Amerigo Vespucci verknüpft ist und der einstmals in Freiburg studierte, wo man auch heute noch diesen frühen Studenten ehrt und ihm gleich auf dem Gebiet der Universität einen Gedenkort eingerichtet hat. Selbst die Werbung für die Stadt Freiburg war nicht untätig, schrieb man doch, dass Amerika in Freiburg entdeckt worden sei. Das ist nun - wie häufig bei Werbung völlig übertrieben und ein wenig maßlos. Aber wahr ist doch so viel, dass es dieser Waldseemüller war, der in einer Gruppe von Gelehrten Männern, die in Saint-Dié zusammenkamen, sich sehr intensiv mit den Nachrichten über die jüngst entdeckten Inseln beschäftigte und bald schon versuchte, in die Kosmographie des Ptolemaeus diese neue Lage der Dinge einzuzeichnen. Ein neues Bild der Welt, ein neues Weltbild war im Entstehen begriffen.

Unsere Beschäftigung und Auseinandersetzung mit einigen frühneuzeitlichen Weltkarten und Kartenwelten soll uns einen Eindruck davon vermitteln, wie dieses neue Weltbild entstand. Es ist beeindruckend, auf welche Weise sich der Horizont der mittelalterlichen Welt weitet, der noch ganz in den Vorstellungen der

1 Für eine ausführlichere Besprechung der so verschiedenartigen Weltkarten vgl. Ette, Ottmar: TransArea. Eine literarische Globalisierungsgeschichte. Berlin - Boston: Walter de Gruyter 2012, S. 53-101.

Ә Open Access. (C) 2020 Ottmar Ette, publiziert von De Gruyter. (cc)BY-NC-ND Dieses Werk ist lizenziert unter der Creative Commons Attribution-NonCommericial-NoDerivatives 4.0 Lizenz.

https://doi.org/10.1515/9783110650686-003 


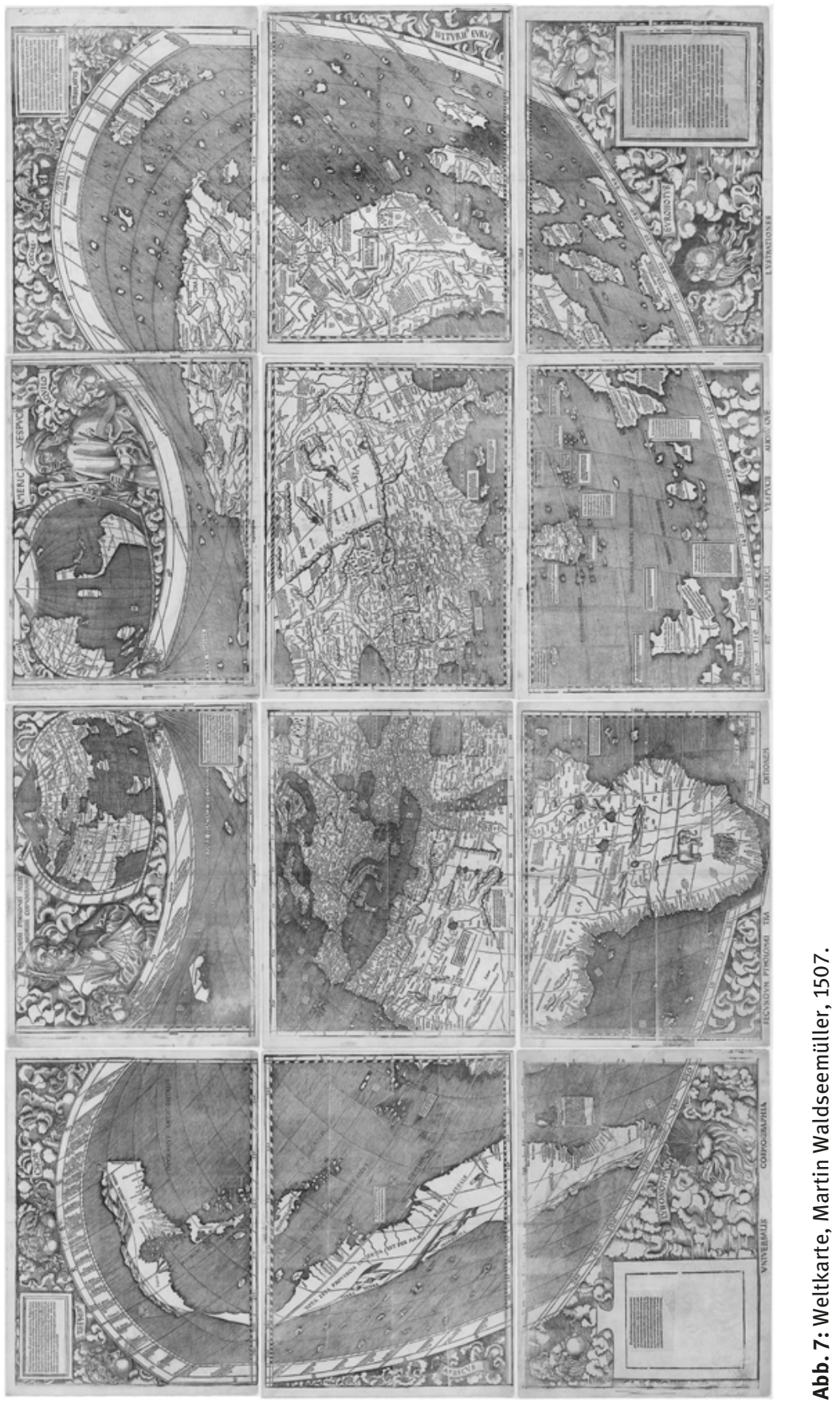




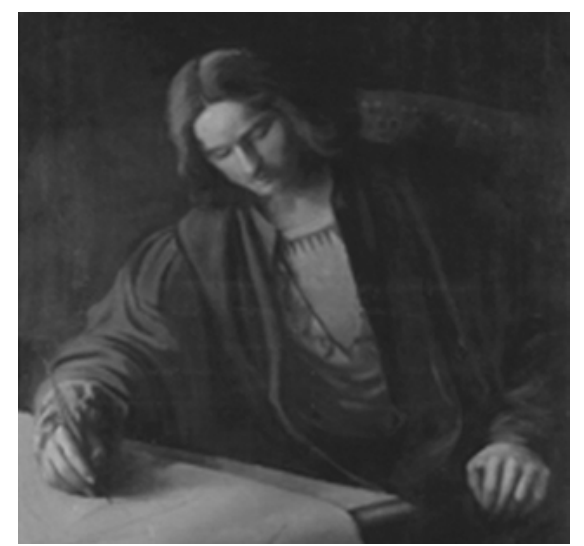

Abb. 8: Martin Waldseemüller (Wolfenweiler, um 1470 - Saint-Dié, 1520).

griechisch-römischen Antike gefangen ist und nicht über deren Grenzen hinausgeht. Dann aber setzt eine schier unablässige Abfolge von Reisen in Weltgegenden ein, die ptolemäisch nicht mehr zu verorten waren. Die Welt beginnt, sich auf dramatische Weise zu verändern - unter dem Eindruck von Reiseberichten, die sich wie in einer wunderbaren Perlenkette auffädeln lassen. Neue Inseln, neue Länder tauchen buchstäblich aus dem Meer auf. Und der Druck dieser „Entdeckungen“, die nur Entdeckungen für die Europäer waren, ist ungeheuer und sprengt die ptolemäisch gebundene Vorstellungswelt. Die Umrisse einer Neuen Welt werden skizziert und verfestigen sich, beginnen, Teil eines europäischen Selbstverständnisses und Weltverständnisses zu werden, das von Grund auf erneuert wird. Es ist nicht die Geburt einer „Neuen Welt“, der wir beiwohnen - denn diese hat es ja für die gesamte Menschheit ebenfalls von Anfang der Besiedelung gegeben -, sondern die Geburt und Entstehung eines neuen Weltbildes, eines neuen Bildes von der Welt, der wir beiwohnen. Einer Welt, deren Umrisse und Konturen, aber auch deren „Inhalte“ sich in der ersten wie der zweiten Phase beschleunigter Globalisierung Stück für Stück herausschälen und konkrete Gestalt annehmen.

Entscheidend war für Waldseemüller nun die Erkenntnis Vespuccis, dass es sich bei diesen Gebieten in der Tat um eine Neue Welt handelte, deren erste Karte im übrigen Juan de la Cosa gezeichnet hat. Dieser Juan de la Cosa war bereits bei der ersten Reise des Columbus in einer verantwortlichen Position mit an Bord gewesen, und er war auch an der ersten Reise jenes Mannes beteiligt, dessen Vorname - lautete er nun Alberrigo oder Amerigo - später dem Kontinent den Namen geben sollte.

Martin Waldseemüller entwarf also ein neues Kartenbild der Welt, das ich Ihnen ebenso wie die Karte des Juan de la Cosa mitgebracht habe und vor Augen führen will. Sehr langsam erst, aber dann mit unglaublicher 
Geschwindigkeit begann sich eine neue Welt herauszuschälen. Und aller Augen richteten sich auf diese Neue Welt, diesen „mundus novus“, von dem nur Christoph Columbus nicht verstehen wollte, dass sie nichts mit Asien und dem Land des Großen Khan zu tun hatte.

Die Karte von Martin Waldseemüller gibt bereits einige Konturen dieser Neuen Welt preis, die wir noch heute deutlich erkennen können. Der ehemalige Freiburger Student übersetzte zudem einige Texte des italienischen Kaufmanns und Seefahrers, wobei nicht alle dieser Texte tatsächlich von Vespucci stammen, ihm aber damals zugeschrieben wurden. Doch mischen wir uns nicht in den Streit ein, welche Texte aus der Feder des Italieners selber und welche aus der Feder eines in der Forschung so bezeichneten Pseudo-Vespucci stammten. Wirklich wichtig ist etwas Anderes für uns. Denn Waldseemüller hatte gute Gründe dafür - zumindest aus seiner Sicht -, in die weiße, leere Fläche des nun entworfenen, erfundenen Kontinents jenen Namen einzuschreiben, den diese freilich heute größer gewordene Fläche noch immer trägt: America. Hören wir also Martin Waldseemüller selbst, wie er Ptolemäus korrigierte:

Jetzt aber sind auch diese Teile schon weiter erforscht und ein anderer vierter Teil ist durch Americus Vesputius - wie man im folgenden hören wird - gefunden worden. Da sowohl Europa als Asien ihre Namen von Frauen erhalten haben, sehe ich nicht, wie jemand mit Recht dagegen sein könnte, diesen Teil nach dem Entdecker Americus, einem Mann von scharfsinnigem Verstand, „Americe“, gleichsam Land des Americus, oder eben „America“ zu benennen.

Dessen Lage und die Sitten des Volkes kann man aus den vier Seefahrten des Americus, die unten folgen, klar erkennen.

So weiß man nun, daß die Erde in vier Teile eingeteilt ist. ${ }^{2}$

Die vier Teile der Welt sind gefunden, les quatre parties du monde oder las cuatro partes del mundo erstmals auf einer Karte mit dem Namen des neuen Kontinents eingetragen. Bei der kartographischen Umsetzung des nun neu entworfenen Bildes von Amerika finden wir bei Waldseemüller - dazu könnte ich noch einige weiterführende Überlegungen anstellen - eine Reihe interessanter und aufschlussreicher Elemente. So handelt es sich bei der Weltkugel nicht um eine wirkliche Kugel, sondern eher um einen Apfel, der an Martin Behaims Erdapfel erinnert. Columbus selbst sprach von einer Birne, der eine Frauenbrust aufgesetzt sei, und eben diese aufgesetzte Frauenbrust

2 Waldseemüller, Martin: Cosmographiae Introductio um quibusdam geometriae ac astronomiae principiis ad eam rem necessariis. Insuper quatuor Americi Vespucii navigationes. Universalis Cosmographiae descriptio tam in solido quam plano, eis etiam insertis, quae Ptholomaeo ignota a nuperis reperta sunt. Saint-Dié, 1507, Kap. IX, zitiert in Bitterli, Urs (Hg.): Die Entdeckung und Eroberung der Welt. 2 Bde. München: C.H. Beck 1980, Bd. 1, S. 43. 
finden wir in gewisser Weise bei Waldseemüller wieder im Bereich des Nordpols, eine von der Formgebung natürlich an den Läuterungsberg erinnernde Gestaltung mittelalterlicher Provenienz. Die Form der Erde: Bis in die Zeiten der französischen Akademiker des 18. Jahrhunderts, welche ihre genauen Längen- und Breitengrade bestimmten, war sie eine ideale Projektionsfläche für die Vorstellungen, Wünsche und Obsessionen der Seefahrer und Kartographen.

Unser obiges Zitat stammt von Seite 43 der von Urs Bitterli herausgegebenen Sammlung Die Entdeckung und Eroberung der Welt, eine Sammlung, die zahlreiche wichtige Dokumente enthält und in der Kurzbibliographie zu finden ist. Dabei erkennen Sie im Übrigen auch, dass Waldseemüller auf die Geschlechterdifferenz eingeht, insoweit Namen von Kontinenten, selbst wenn sie sich wie in diesem Fall von Männern herleiten, einen weiblichen Charakter tragen müssen. Das ist durchaus nicht uninteressant, denn in der Tat wird hier die Geschlechtlichkeit zugleich dienstbar gemacht für einen Akt männlicher Benennung, eine Erstellung einer bestimmten Serie von Erdteilen und die Übertragung des Vornamens - und nicht etwa des Nachnamens. Ersterer konnte im Übrigen vielleicht sogar doch noch einen amerikanischen Bezug haben, wäre es doch durchaus möglich, dass Vespucci ursprünglich Alberrigo hieß und er später seinen Namen veränderte, als er im mesoamerikanischen Sprachraum mit einem indigenen Ausdruck in Verbindung kam, welcher mit dem Wind verbunden ist: Amerika. Aber lassen wir diese Spekulationen.

Wie dem auch immer sei: Las Casas kam mit seinem Vorschlag zu spät, den „neuen“ Kontinent nach seinem „eigentlichen“ Entdecker zu benennen, wenn dies auch später dann zumindest einer der sich zu Beginn des 19. Jahrhunderts bildenden Nationalstaaten tat und den Namen dessen wählte, der am Beginn der europäischen Kolonialgeschichte stand: Ich meine natürlich das Land Kolumbien. Da ist es nur zu verständlich, dass es im selben Zeitraum - oder nur wenig später - dann ein anderes Land dieses Kontinents geben sollte, das den Namen jenes Mannes verewigte, der diesem kolonialen Zustand zumindest im politischen Bereich ein Ende setzte: Bolívar und sein Bolivien. Das Schöne und Gemeinsame ist dabei, dass beide in der Tat mit den jeweiligen Ländern, die ihre Namen tragen, herzlich wenig zu tun haben. Sie sehen schon: Die Namensgebungen sind weithin von Zufälligkeiten und Vorlieben abhängig - sie erläutern nichts, sondern verweben und vermischen, wie es die Mythen tun.

Nun aber zur Tradition des Insel-Buches, das lange vor der sogenannten Entdeckung Amerikas bestand und in der Folge völlig neu konzipiert wurde. Vierzig Jahre nach Erscheinen des Isolario des Venezianers Bartolomeo dalli 
Sonetti, ${ }^{3}$ ließ der ursprünglich aus Padua stammende Benedetto Bordone, ebenfalls in Venedig, im Jahre 1528 ein weiteres Insel-Buch erscheinen.

Mit diesem Buch, das in einen Zeitraum sich deutlich intensivierender literarischer Insel-Produktionen fällt, für die Thomas Morus' Utopia (1516) stellvertretend stehen mag, ${ }^{4}$ gilt es, sich etwas ausführlicher zu beschäftigen - und dies nicht allein, weil zum selben Zeitpunkt, also für das Jahr 1528, gerade im Englischen die ersten Belege für ein Reden von „der ganzen Welt“ (the whole world) nachgewiesen sind. ${ }^{5}$ Denn der Erscheinungszeitpunkt von Bordones Isolario liegt nicht nur nach der sogenannten „Entdeckung“ Amerikas, sondern auch nach der Eroberung der Hauptstadt des Aztekenreiches durch die Spanier unter Hernán Cortés - und damit nach der ersten dauerhafteren Konfrontation mit amerikanischen Hochkulturen. Diese Erfahrungen gehen zweifellos in Benedetto Bordones europäischen Weltentwurf mit ein.

Vor dem Hintergrund dieser nicht zuletzt auch kulturellen Herausforderungen für das Selbstverständnis der Europäer kann dieses überaus erfolgreiche Werk für sich in Anspruch nehmen, anders als Bartolomeo dalli Sonettis Isolario nicht nur einen Teil des Mittelmeeres, sondern eine ganze Welt von Inseln in weltweiter Projektion entworfen zu haben. Die Welt erscheint auf diesen Karten als eine Welt von Inseln, als eine Welt in Stücken. In Bordones Isolario stoßen wir auf eine frühe Antwort ebenso auf die erste Phase beschleunigter Globalisierung wie auch auf all jene Problematiken, die aus der Frage nach der Konvivenz mit einer unbestreitbaren Vielfalt an Religions-, Gesellschafts- und Gemeinschaftsformen entstanden. Die unterschiedlichen Bewohner der Welt, der Erde, bewohnen allesamt verschiedenartige Inseln mit einer je eigenen Geschichte und Kultur, eigenem Klima, eigener Agrikultur, Wirtschaft und Gesellschaft. Alles steht zunehmend mit allem in Verbindung und entwickelt relationale Beziehungen in einem Gefüge, das sich aus unterschiedlichen Perspektiven unterschiedlich darstellt. die Welt ist eins und zugleich diskontinuierlich aufgebaut.

Was aber genau fand sich in Benedetto Bordones Isolario verzeichnet? Und warum ist dieses Insel-Buch, das die unterschiedlichsten Reisen verzeichnet, für uns so wichtig? Der wohl um 1460 geborene „Intellektuelle aus

3 Vgl. Dalli Sonetti, Bartolomeo: Isolario. Venedig 1485. With an Introduction by Frederick R. Goff. Amsterdam: Theatrum Orbis Terrarum Ltd. 1972.

4 Vgl. hierzu Billig, Volkmar: Inseln. Geschichte einer Faszination. Berlin: Matthes \& Seitz, S. 81-88.

5 Vgl. hierzu Connor, Steven: 'I Believe That the World'. In: Nünning, Vera / Nünning, Ansgar / Neumann, Birgit (Hg.): Cultural Ways of Worldmaking. Media and Narratives. Berlin New York: Walter de Gruyter 2010, S. 30. 


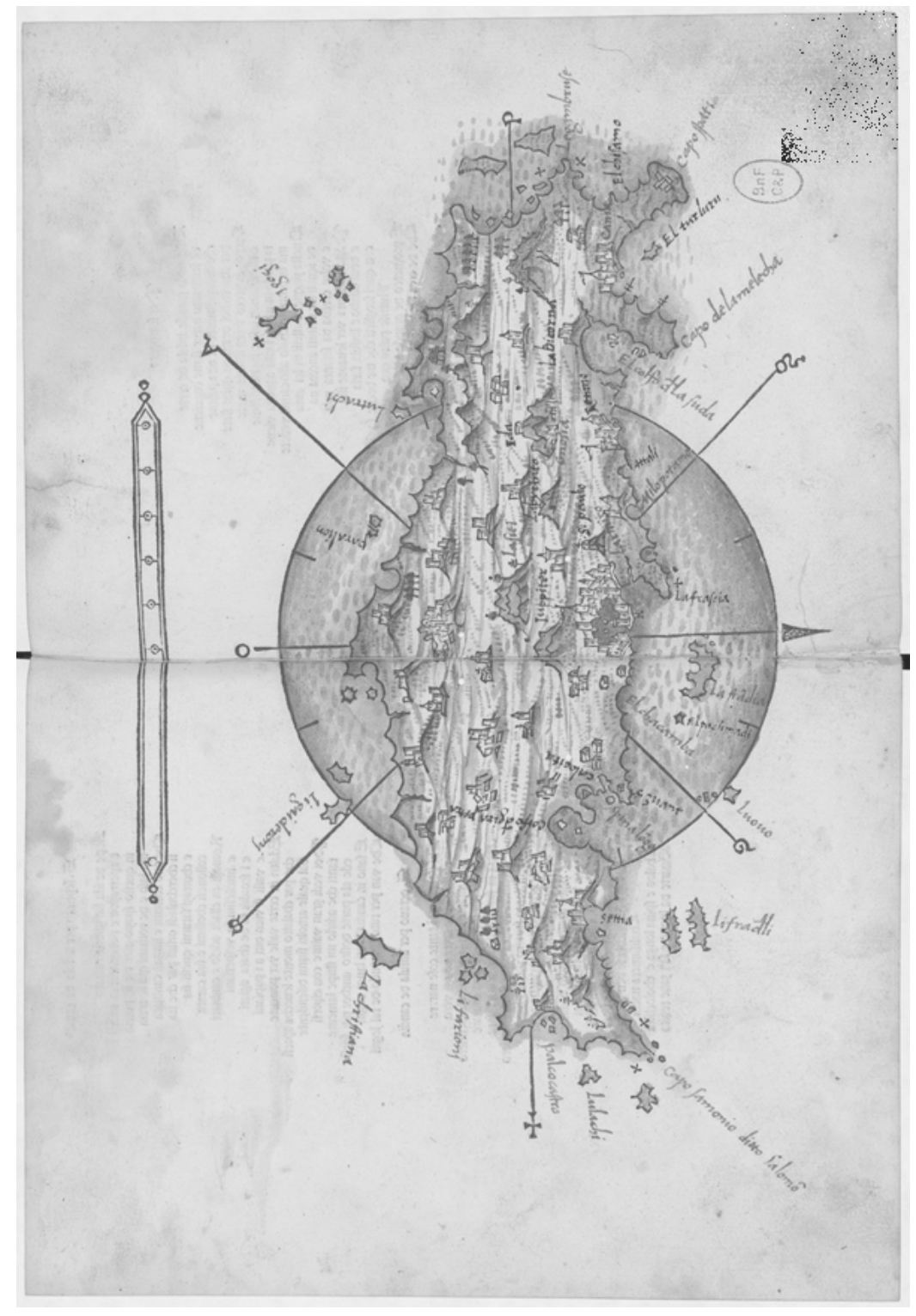

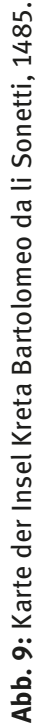


Padua“, 6 der am 10. April 1539 in Venedig verstarb und dessen unehelicher Sohn Scaliger eine der bekanntesten Figuren des europäischen Humanismus werden sollte, ${ }^{7}$ veröffentlichte 1528 die Erstausgabe seines Insel-Buches unter dem recht ausführlichen Titel Libro di Benedetto Bordone nel qual si ragiona de tutte l'isole del mondo, con li lor nomi antichi \& moderni, historie, favole, \& modi del loro vivere \& in qual parte del mare stanno, \& in qual parallelo \& clima giacciono. ${ }^{8}$ Nachfolgende Editionen, die noch zu Lebzeiten des Autors ebenfalls in Venedig erschienen, trugen seit 1534 den bündigeren Titel Isolario, der sich mit Bezug auf dieses Werk auch weitgehend in der (im Übrigen nicht sehr intensiven) Forschung durchgesetzt hat. Daher soll im folgenden Bordones Libro als Isolario bezeichnet und damit deutlich auf die skizzierte venezianische Gattungstradition des Insularium bezogen werden. Dieses Insularium aber ist zugleich ein Imaginarium, das die Welt als eine Inselwelt entwirft.

Beschäftigen wir uns kurz mit dem Aufbau des Bandes. Bordones Insel-Buch besteht aus drei schon in ihrem Umfang sehr ungleichen Teilen, deren erster mit insgesamt neunundzwanzig Karten die atlantische Inselwelt einschließlich der Ostsee enthält, deren zweiter mit dreiundvierzig Karten die Inseln des Mittelmeers beleuchtet, und deren dritter mit nur mehr zehn Karten die Inseln des Fernen Ostens aufruft. Gewiss, das ist ein Ungleichgewicht; aber selbstverständlich ist dieses Unverhältnis der damaligen Informationslage in Venedig geschuldet. Dabei versucht Bordone, in einer sich im Aufbau wiederholenden Abfolge gleichsam wissenschaftlich geordnet Informationen zur geographischen Lage, zu Klima und Geschichte, zur Bevölkerung, zu Fauna oder Flora und vielen weiteren Aspekten von allgemeinem Interesse für seine europäische Leserschaft zu geben. Dabei war Venedig zum damaligen Zeitpunkt ein Kreuzungspunkt europäischer Informationen über die außereuropäische Welt, so dass wir durchaus von einem Informationsstand sprechen, der insgesamt bei weitem überdurchschnittlich war.

\footnotetext{
6 Serafin, Silvana: Immagini del mondo coloniale nella cultura veneziana dei secoli XVI eXVII. In: Rassegna Iberistica (Venedig) 57 (Juni 1996), S. 39-42, hier S. 39.

7 Vgl. hierzu Karrow, Robert W.: Benedetto Bordone. In (ders.): Mapmakers of the Sixteenth Century and their Maps. Chicago: Speculum Orbis Press 1993, S. 89.

8 Bordone, Benedetto: Libro di Benedetto Bordone nel qual si ragiona de tutte l'isole del mondo, con li lor nomi antichi \& moderni, historie, favole, \& modi del loro vivere \& in qual parte del mare stanno, \& in qual parallelo \& clima giacciono. Con il breve di papa Leone. Et gratia \& privilegio della Illustrissima Signoria com' in quelli appare. Vinegi [Venezia]: per Nicolo d'Aristotile, detto Zoppino 1528. Im folgenden beziehe ich mich auf diese Ausgabe, die überdies als elektronische Fassung 2006 im Harald Fischer Verlag in Erlangen erschien. Die Übersetzung des Titels ins Deutsche könnte lauten: „Buch des Benedetto Bordone, worin von allen Inseln der Welt berichtet wird, mit ihren alten \& modernen Namen, ihren Geschichten, Erzählungen \& Arten ihres Lebens \& in welchem Teil des Meeres sie sind \& unter welchem Breitenkreis \& Klima sie liegen."
} 
Was alles enthielt dieses Werk, das zweifellos den Informationsstand seiner Epoche spiegelte? Schematische Zeichnungen zur Gradeinteilung der Erdkugel (die ohne die zeitgenössischen Diskussionen um Ptolemäus' kartographische Projektionen gewiss nicht so umfangreich ausgefallen wären), Angaben zu den Wendekreisen sowie zur Schiefe der Ekliptik des Globus, zur Segmentierung der Windrose in Antike und Gegenwart, aber auch Überblickskarten von Europa, dem östlichen Mittelmeer sowie der gesamten zum damaligen Zeitpunkt bekannten Welt runden Bordones Isolario ab und vermitteln dem zeitgenössischen Leser und darin dürfte ein Gutteil der Attraktivität des Werkes gelegen haben - ein ebenso anschauliches wie farbenfrohes Bild von unserem Planeten. Das gesamte Werk ist auf Vielfalt und Diversität angelegt. Gerade die ,wissenschaftliche“ Rahmung signalisiert den Anspruch des Isolario, seinen Betrachtern und Lesern verlässliche, faktenbezogene Informationen und damit ein „wahres“ Wissen über die unterschiedlichsten Weltteile zukommen zu lassen. Translokale Bezüge etwa zwischen unterschiedlichen Inseln desselben Archipels runden die jeweiligen Textteile $\mathrm{ab}$ und sorgen dafür, dass sich die Betrachter auch ein Bild von den wechselseitigen archipelischen Wirtschafts- und Kulturbeziehungen machen konnten.

Vergleicht man unter diesem Gesichtspunkt Benedetto Bordones Weltkarte $^{9}$ von 1528 mit jener des Spaniers Juan de la Cosa aus dem Jahre 1500 (vgl. Abb. 15: Mapamundi, Juan de la Cosa, circa 1500) so zeigt sich zum einen zwar deutlich, wie sehr die europäischen Kartennetze nun den gesamten Planeten erfassen und in die gleiche Spatialität und Temporalität integrieren beziehungsweise zwingen. Bordone erweist sich hier als ein Kartograph, der sich der wesentlich von Florenz ausgehenden Diskussionen um die Perspektive bewusst ist und diese in sein eigenes Kartenbild zu integrieren versteht. Der Maler und Miniaturist aus Padua wusste, auf welchem Stand sich die zeitgenössische Kartenkunst und Malerei befand.

Zum anderen aber wird deutlich, dass die im Vergleich zu Juan de la Cosa wesentlich geringere Präzision des venezianischen Isolario auf eine andere Ausrichtung und Zielsetzung dieses Inselbuches hindeutet. Gewiss: Die spanische Karte von 1500 war nicht für ein größeres Publikum, sondern für sehr begrenzte politische und militärische Eliten im spanischen Kolonialsystem bestimmt. Sie war eine Geheimkarte, die sicherlich nur wenige

9 Ein Abdruck dieser Karte findet sich im „Unsichtbaren Atlas“ in der bereits genannten Edition von Humboldt, Alexander von / Ette, Ottmar (Hg): Kritische Untersuchung zur historischen Entwicklung der geographischen Kenntnisse von der Neuen Welt und den Fortschritten der nautischen Astronomie im 15. und 16. Jahrhundert [...], 2 Bde., Frankfurt am Main/ Leipzig: Insel Verlag, 2009, Bd. 2, Abb. 28. 


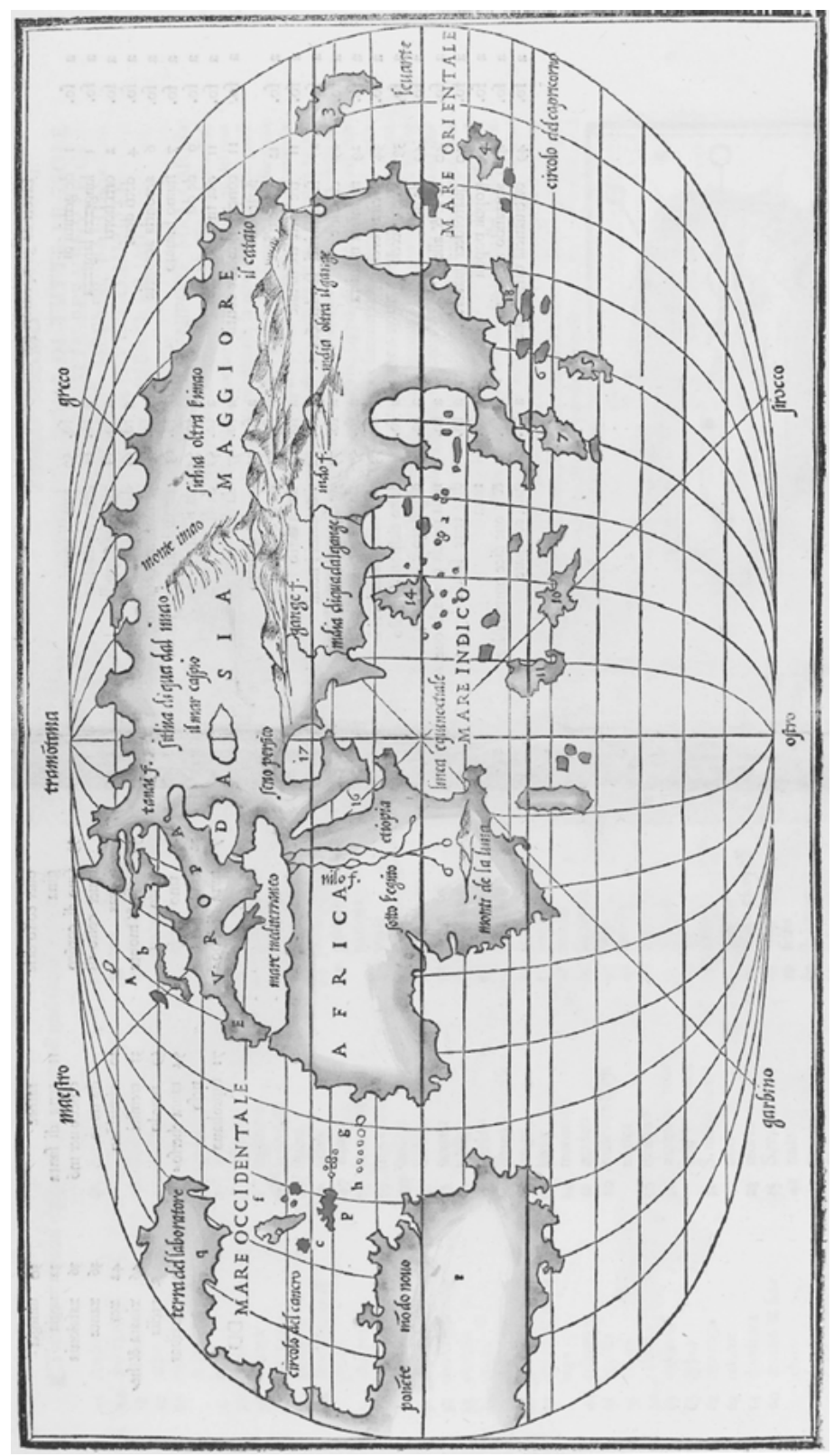

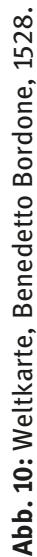


Eingeweihte $\mathrm{zu}$ Gesicht bekamen. Zugleich repräsentiert sie das Herrschaftswissen einer Weltmacht, die über detaillierte, aber geheim zu haltende Informationen verfügt, ohne die nachfolgende Eroberungen ferner Weltteile gar nicht möglich gewesen wären. Daneben vermittelte sie den Katholischen Königen erstmals eine visuelle Anschauung von jenen Gebieten, die in Übersee an ihre Krone gefallen waren und nunmehr zu ihrem Herrschaftsbereich gehörten.

So kommen der spanischen Weltkarte gänzlich andere Funktionen und Aufgabenbereiche zu. Juan de la Cosas Karte ist folglich direkt in eine Pragmatik eingebunden, in der das vor Ort Vorgefundene, das in einer anderen Zeit und in einem anderen Raum Erfundene sowie das eigene Erlebte in eine nautisch-militärische Zielsetzung integriert werden, die man mit Fug und Recht als expansionistische Weltpolitik der spanischen Krone in Konkurrenz zu anderen Weltmächten (und insbesondere der Seemacht Portugal) bezeichnen muss. Sie ist imperial gedacht und imperial gemeint. Doch auf diese Karte von Juan de la Cosa kommen wir erst noch zurück.

Dies ist in Benedetto Bordones Isolario in weitaus geringerem Maße der Fall, auch wenn die Interessen der Handelsmacht Venedig in allen Teilen dieses Weltentwurfes spürbar sind. Doch Ausgangslage und Funktionen des Isolario waren im damaligen Venedig gänzlich andere. In Bordones Insularium stößt man im Vergleich mit der spanischen Weltkarte von 1500 nicht nur auf zahlreiche Ungenauigkeiten, sondern auch auf eine Vielzahl an Widersprüchen, die sich dem aufmerksamen Blick des Betrachters rasch enthüllen. Worin liegen diese Widersprüche zwischen den einzelnen Kartenbildern im selben Isolario begründet?

Vergleicht man etwa die Einzelkarte der Insel $\mathrm{Cuba}^{10}$ aus dem ersten Teil mit jenen Inseln der Karibik, die auf der Weltkarte desselben Bandes eingezeichnet sind, so erkennt man leicht, dass sich die Zeichnung der Umrisse Cubas - die nicht das Geringste mit der Genauigkeit bei Juan de la Cosa achtundzwanzig Jahre zuvor zu tun hat - nirgendwo wiederfinden lässt. Ganz offenkundig ist Benedetto Bordone die Karte des Juan de la Cosa nicht zugänglich gewesen. Auf der separaten Karte von Cuba erscheint bei Bordone eine Insel, deren im Grunde

10 In der von ihr kuratierten Ausstellung 'Faszination Kuba', die erstmals an der Württembergischen Landesbibliothek Stuttgart vom 7. März bis 19. Mai 2007 gezeigt und am 23. April 2009 an der Universität des Saarlandes in Saarbrücken wiedereröffnet wurde, hat Birgit Oberhausen die Bedeutung dieser Karte zurecht hervorgehoben. Vgl. den Ausstellungskatalog von Oberhausen, Birgit: Faszination Kuba in der Landesbibliothek: Literatur und Kultur 1492-2006. Stuttgart: Württembergische Landesbibliothek 2007. 


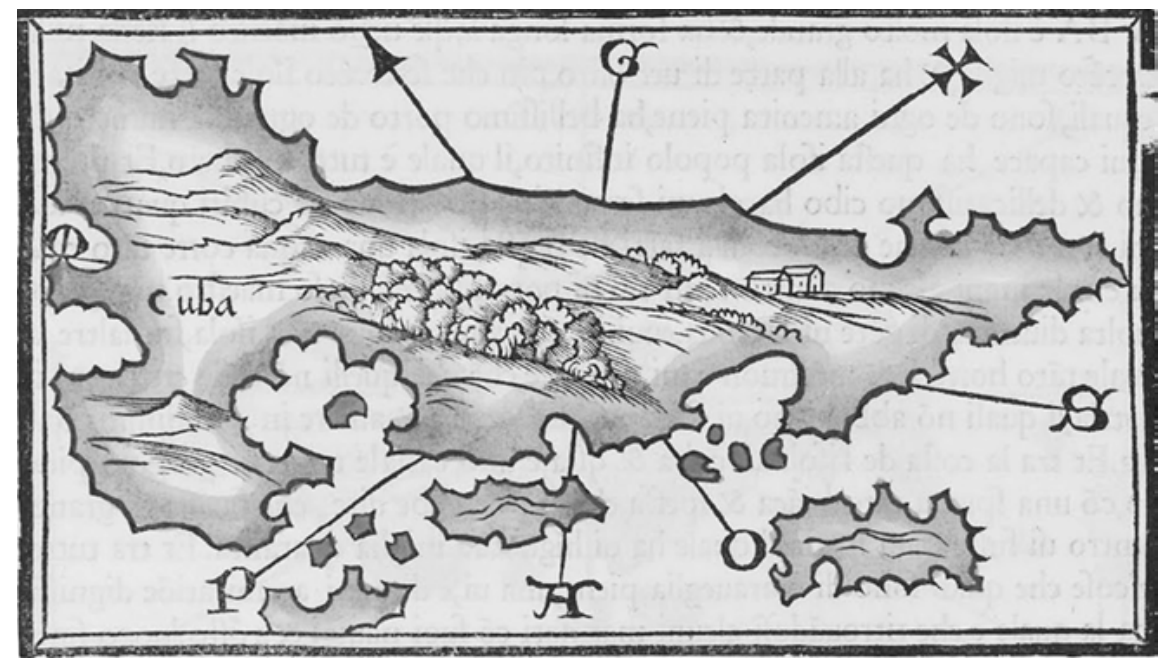

Abb. 11: Karte der Insel Kuba, Benedetto Bordone, 1528.

schematisiert und austauschbar gezackte und gebuchtete Küstenverläufe das Kartenbild eines Eilands erfinden, in dessen Binnenraum Gebirge und Hügel, Wälder und Äcker, aber auch italienisch anmutende Gehöfte zu sehen sind. Die erste kartographische Einzeldarstellung von Kuba bietet das Bild einer erfundenen, einer imaginierten Insel.

Dominiert folglich das Imaginarium im Insularium? Es wäre zu einfach, wollte man Bordone hier der Lüge bezichtigen und seinem Werk jedwede Glaubwürdigkeit bestreiten. Denn das Insularium entpuppt sich hier zugleich als ein Imaginarium, das sich an den zugänglichen Informationen ausrichtet. Auf durchaus andere, mit Juan de la Cosa nur begrenzt vergleichbare Weise gehen Finden und Erfinden bei Bordone Hand in Hand, entwerfen zugleich aber auch eine Welt, in der in den Begleittexten die unterschiedlichsten menschlichen Lebensformen und Lebensnormen kopräsent sind und entfaltet werden. Denn gerade die Differenz in Fragen der Konvivenz wird im Isolario immer wieder in den Vordergrund gestellt. Kein Zweifel: Es geht bei Bordone immer wieder um die Formen und die Normen menschlichen Zusammenlebens in einer an Diversität reichen Welt, in welcher die Menschen auf jeweils eigenen Inseln leben.

Auch wenn die in Benedetto Bordones Isolario integrierte Weltkarte versucht, ein Gesamtbild der Erde gleichsam als 'Totaleindruck', auf einen Blick, zu ermöglichen, weisen die zahlreichen Abweichungen zwischen den separaten Insel-Karten und der 'vollständigen' Gesamtkarte doch darauf hin, dass der Schwerpunkt dieses Werkes von 1528 darauf gelegt wurde, die Dimension des Weltweiten nicht aus 
einer homogenisierenden Perspektive zu betreiben, sondern naturgeschichtliche, klimatische, historische und kulturelle Diversität plastisch vor Augen zu führen. Bordones Weltkarten und sein gesamtes Insularium zielen nicht auf eine kontinentale Homogenität, sondern auf eine unverkennbare Diskontinuität unterschiedlicher Lebensformen und Lebensnormen. Darin, so scheint mir, liegt die Besonderheit der dieser transmedialen Gattung eingeschriebenen Traditionslinie. Denn sie beharrt auf Polyperspektivität und auf einer grundlegenden Diversität aller Lebensbereiche.

Diese Anlage wird bereits im ersten, transatlantischen Teil in aller Deutlichkeit sichtbar. An die jeweils mit ausführlichen Textteilen versehenen Karten von Island, Irland, Südengland, von der Bretagne, Nordwestspanien und Skandinavien schließen sich die nicht weniger textuell eingebetteten Karten von Nordamerika und des Nordatlantik, der Stadt Temistitan (also Tenochtitlán, das spätere Mexico), von Zentral- und Südamerika, Hispaniola, Jamaica, Cuba sowie weiterer karibischer Inseln an, bevor wir über Porto Santo, Madeira, die Kanarischen Inseln, die Kapverden und die Azoren wieder die Bucht von Cádiz in Südspanien und damit die Alte Welt in einem sich rundenden Kreis erreichen. Bereits die Nennung der Stationen dieser transatlantischen 'Rundreise' demonstriert, dass wir es hier nicht nur mit im traditionellen Sinne in Gänze von Wasser umschlossenen Inseln zu tun haben, sondern Teile von Kontinenten einbezogen sind, auch wenn nicht alle dieser zuletzt Genannten bereits für die Zeitgenossen als Kontinente erkennbar und bekannt sein konnten. So ergibt sich ein den Atlantik umspannender Reigen von größeren und kleineren Inseln, die stets voneinander getrennt sind und ihr Eigen-Leben besitzen. Aufgrund der nicht flächigen, sondern punktuellen Vorgehensweise entsteht ein translokales Beziehungsgeflecht der Differenz, das weltumspannend angelegt ist. Die ganze Welt erscheint als eine Inselwelt.

Offenkundig ist, dass die nicht nur mit Blick auf Labrador, Zentralamerika, Mexico oder Südamerika, sondern auch auf Skandinavien, das spanische Galizien oder die kontinentaleuropäische Bretagne unstrittig überaus weite Fassung des Begriffes 'Insel' eine Welt modelliert, die sich aus den verschiedenartigsten Lagen und Größen, Formen und Figuren von Inseln zusammensetzt. Entscheidend ist dabei, dass es keine territorialen Kontinuitäten gibt in diesem Entwurf einer Welt in Stücken. Mithin dominiert nicht eine kontinentale, das heißt zusammenhängende, kontinuierliche Sichtweise der Welt - wie sie durch die untereinander zusammenhängenden altweltlichen Kontinente Asien, Europa und Afrika nahegelegt wird -, sondern eine hochgradig diskontinuierliche und fragmentierte Weltsicht, die eine gleichsam zersplitterte, in einzelne Scherben zerborstene Welt vor Augen führt. 
Es ist - um es mit einer Formel von Clifford Geertz ${ }^{11} \mathrm{zu}$ sagen - wahrhaftig eine Welt in Stücken: eine höchst komplex in Inseln zerstückte Welt, die nur sehr schwer einer einzigen Macht zu unterwerfen und in eine menschheits- oder heilsgeschichtliche Kontinuität zu bringen ist. Denn jede Insel ist zwar Teil einer zusammenhängenden Welt, eröffnet aber eine je besondere Perspektive auf diesen definitiv zur Erdkugel gerundeten Planeten. $\mathrm{Zu}$ groß sind die Unterschiede zwischen den Geographien, zu bedeutend die Diversität der Kulturen, zu auseinanderstrebend die Vielheit der Sprachen, als dass sich eine einfache Einheit der Menschheit in diesem Entwurf abzeichnen würde.

Dass sich die Insularien gerade in der venezianischen Welt entwickelten und zu einer eigentlichen 'Spezialität' der Lagunenstadt wurden, ${ }^{12}$ ist sicherlich nicht dem Zufall geschuldet. Es ist die Lagunenstadt selbst, die für den Erdkreis gleichsam das Modell geliefert hat. In Bordones Libro oder Isolario läßt sich die Sonderstellung Venedigs im weltweiten Maßstab bereits auf den ersten Blick erkennen. Denn der auf Pfählen errichteten Stadt ist - neben Einzelkarten zu den Inseln Murano oder Mazorbo - ein besonders liebevoll und mit $230 \times 326 \mathrm{~mm}$ kaum kleiner als die Weltkarte ausgeführter Stadtplan beigegeben, der die Inselstadt mit ihrem Lido und dem Festland als das Zentrum eines Archipels entwirft, ${ }^{13}$ in welchem alle Inseln miteinander in Beziehung stehen. Urbi et orbi: Wir haben es mit einer Stadt als Mikrokosmos einer ganzen Welt zu tun. Stadt und Welt werden hierbei als Inseln perspektiviert und als Fraktal in Szene gesetzt. Die Stadt der Inseln ist das Modell für eine Welt von Inseln.

Aus diesem Blickwinkel der Insel-Stadt aber wird deutlich, warum im ersten Teil Tenochtitlán, La gran citta di Temistitan, als Hauptstadt der Azteken eine besonders herausgehobene Stellung zuerkannt wird. Zwar blieb der Plan des späteren Mexico-Stadt mit einer Größe von $164 \times 163 \mathrm{~mm}$ deutlich kleiner als der Plan Venedigs; doch waren die der Hauptstadt Moctezumas gewidmeten und vielfach auf die zeitgenössischen, insbesondere von Nicolò Liburnio ${ }^{14}$ ausgearbeiteten Darstellungen zurückgreifenden Seiten, in welchen die Cartas de relación beziehungsweise deren Verfasser Hernán Cortés explizit genannt wurden, überaus umfangreich. ${ }^{15}$ Kein Zweifel: Tenochtitlán war für

11 Vgl. Geertz, Clifford: Welt in Stücken. Kultur und Politik am Ende des 20. Jahrhunderts. Aus dem Englischen übersetzt von Herwig Engelmann. Wien: Passagen Verlag 1996.

12 Karrow, Benedetto Bordone, S. 93.

13 Bordone, Benedetto: Libro, Bl. XXX.

14 Vgl. Liburnio, Nicolò: La preclara narratione di Ferdinando Cortese della nuova Hispagna del mare Oceano. Venetia: Bernardino de Viano 1524. Vgl. hierzu Serafin, Immagini del mondo coloniale, S. 40.

15 Bordone, Libro, Bl. X. 
Bordone mehr als ein Klein-Venedig, als ein 'Venezuela', in welchem sich die Grundstruktur der Lagunenstadt spiegelte. Was Venedig in den Augen von Bordone für die Alte Welt, das war die Hauptstadt der Azteken für die Neue Welt, die nicht in ihrer Kontinentalität, sondern in ihrer Inselhaftigkeit präsentiert wurde.

Mit größter Hochachtung, Sorgfalt und Bewunderung werden im Isolario die 'wunderbaren Besitztümer'16 der in der Tat schon zum damaligen Zeitpunkt größten Stadt des amerikanischen Kontinents geradezu besungen. Und zugleich wird bei allen ebenfalls markierten Unterschieden - etwa zwischen der Lage in einem salzigen Meer oder einem Süßwassersee in den Bergen, zwischen unterschiedlichen Klimaten in der Alten und der Neuen Welt etc. doch das Phänomen herausgestellt, dass Tenochtitlán wie Venedig auf unzähligen kleinen Inseln aufruht, was beide in spezifischer Weise als Inselstädte ausweist, die aus und auf einer Vielzahl von Inseln und Inselchen erbaut sind. So erscheint die Totalität einer Welt gerade in ihrer Zerstückung, in ihrer Aufspaltung in unendlich viele Inseln und Inselchen. In diesem Sinne sind sie InselInseln, ${ }^{17}$ denen innerhalb einer transarchipelischen Welt eine herausgehobene Machtstellung mit besonderen Machtansprüchen zukommt, bilden sie doch Fraktale einer Welt als Archipel: basierend auf archipelischen und transarchipelischen Beziehungen. Man könnte hier sehr wohl von einer transarealen Weltsicht in der Frühen Neuzeit sprechen, werden hier doch verschiedenartige geographisch-kulturelle Areas in ihrer Vielbezogenheit wie in ihrer Multiperspektivität gezeigt.

Benedetto Bordones Isolario entfaltet in diesem Sinne eine vielgestaltige Welt, in welcher die Aufspaltung in eine ungeheure Zahl an Inseln jede einzelne dieser für sich isolierten Einheiten als eine Insel-Welt mit jeweils spezifischen Charakteristika repräsentiert. Der literarische Reisebericht ist hier seiner Kontinuität und Linearität enthoben und in eine Vielzahl von Diskontinuitäten aufgelöst. Keine dieser Inseln ist auf eine andere reduzierbar: Alle besitzen sie im globalen Gitternetz mit Blick auf ihre Koordinaten, ihr Klima oder ihre Geschichte, hinsichtlich ihrer Sprachen, Sitten und Gebräuche eine Eigenständigkeit, die sie - man würde im gegenwärtigen Bürokratendeutsch von einem 'Alleinstellungsmerkmal' sprechen - von allen anderen Inseln

16 Vgl. hierzu Greenblatt, Stephen: Marvelous Possessions: the wonder of the New World. Oxford: Clarendon Press 1992.

17 Vgl. hierzu meinen Beitrag Insulare ZwischenWelten der Literatur. Inseln, Archipele und Atolle aus transarealer Perspektive. In: Wilkens, Anna E. / Ramponi, Patrick / Wendt, Helge (Hg.): Inseln und Archipele. Kulturelle Figuren des Insularen zwischen Isolation und Entgrenzung. Bielefeld: transcript Verlag 2011, S. 13-56. 


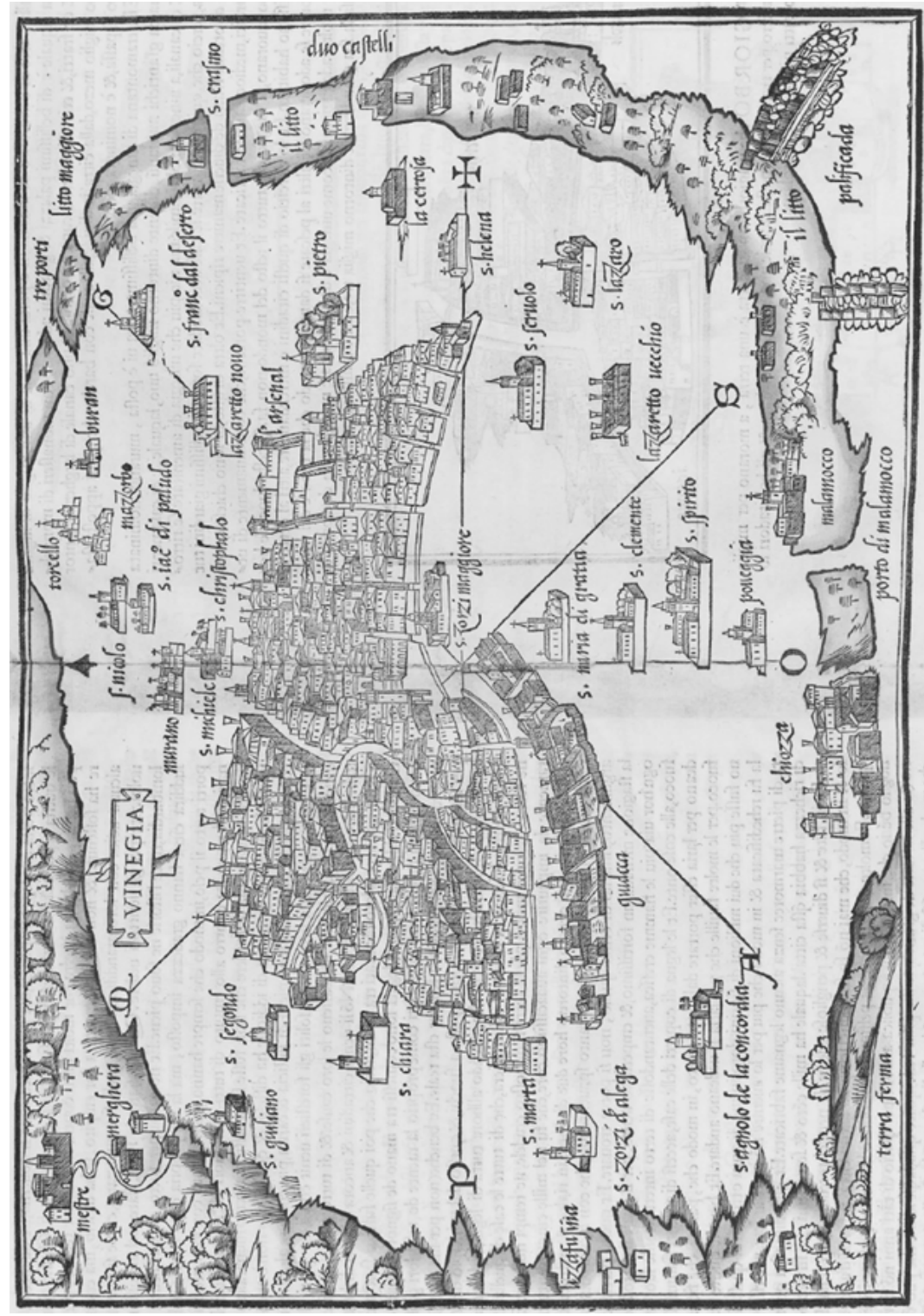

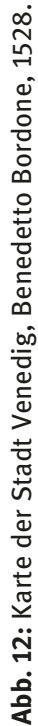




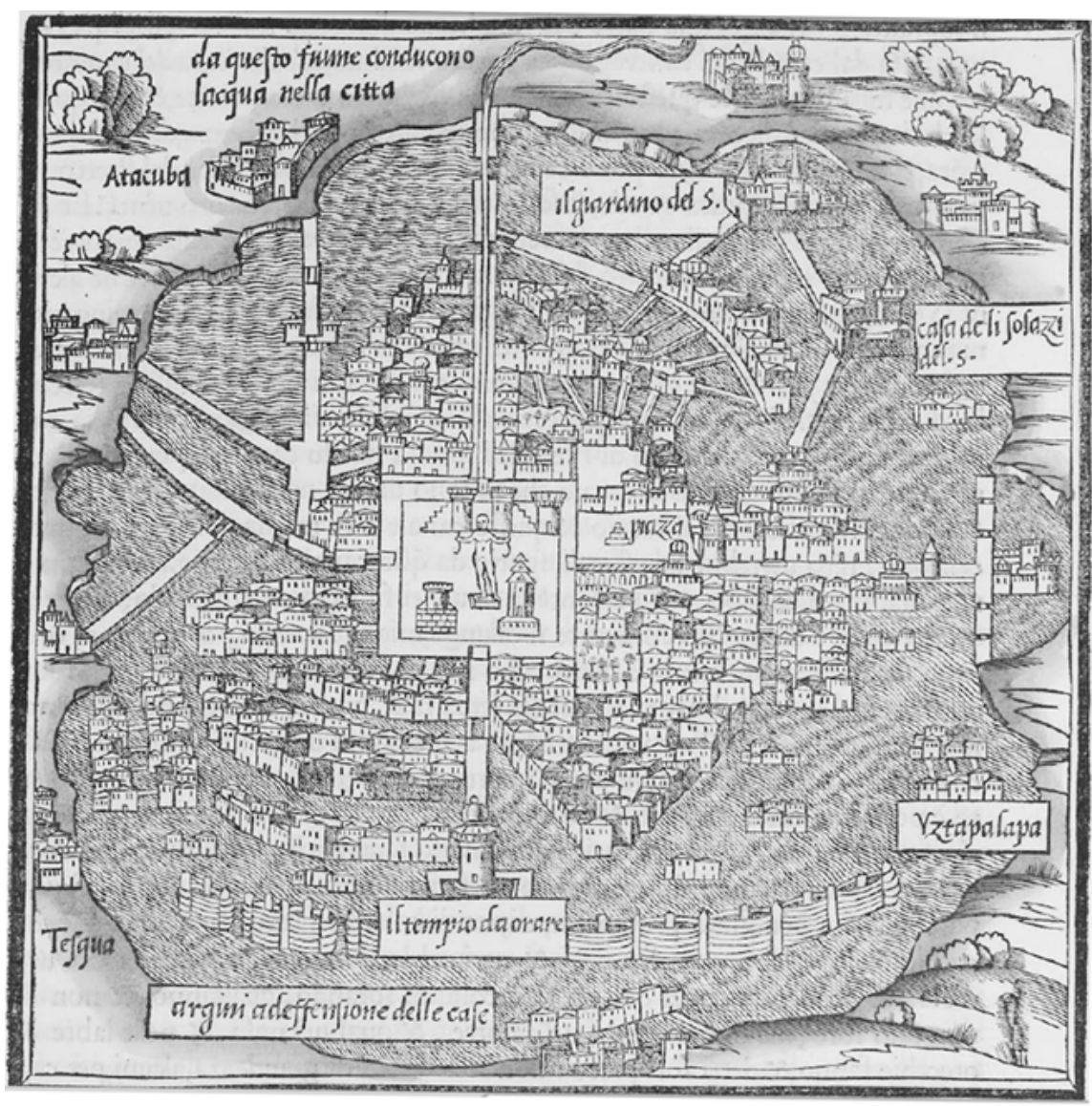

Abb. 13: Karte der Stadt Temistitan [Tenochtitlan], Benedetto Bordone, 1528.

unterscheidet und abhebt. Auch wenn nicht wenige dieser Inseln Teile größerer Archipele sind, werden ihre Verbindungen untereinander doch als sehr begrenzt dargestellt, so dass die Eigen-Logik dieser Insel-Welten mit ihren separaten Eilanden immer wieder unverkennbar hervorsticht.

Doch es gibt neben dieser Logik der Insel-Welt noch eine andere, deutlich gegenläufige Logik. Denn wenn jede Insel nicht nur eine für sich abgeschlossene Welt, mithin eine Insel-Welt, sondern zugleich eine durch vielfache Relationen ausgezeichnete Inselwelt darstellt, dann zeigt sich in diesem Isolario sehr rasch, dass Benedetto Bordone die Eigenständigkeit zuungunsten der Relationalität oder der Relationierbarkeit deutlich privilegierte. 
Der Insel als ganzer Welt tritt hier die ganze Welt als Raum voller Inseln entgegen. Dahinter aber, so steht wohl zu vermuten, verbirgt sich letztlich ebenfalls ein Machtanspruch: jener nämlich, von Europa aus eben jene Vielverbundenheit und Relationalität herstellen zu können, die in der bisherigen Geschichte zwischen diesen Inseln noch nicht bestanden habe. Venedig erscheint als die im eigentlichen Sinne translokale und vielleicht mehr noch translokalisierende Kraft, deren Politik und Handelsmacht die Welt in dieser ersten Phase beschleunigter Globalisierung zu verändern vermag. In Bordones Isolario greift die Stadt Venedig auf jene Archipelisierung zurück, kraft derer sie ihre Macht über andere Areas und Regionen der Welt auszudehnen bemüht ist. Der Isolario lässt sich als Reisebericht lesen, der von der potentiellen Relationierbarkeit und Abhängigkeit einer ganzen Welt verschiedenartiger Inseln im nicht-kontinuierlichen Gestus erzählt.

Venedig aber ist bei Bordone in Lauerstellung. Denn welche Macht wäre besser für die Herstellung weltweiter Relationalität geeignet als eine Seemacht, deren Hauptstadt selbst aus ungezählten Inseln besteht und deren Handelsmacht sich auf eine der größten Flotten der Welt zu stützen vermag, eine Seemacht, die jederzeit in der Lage wäre, zwischen den Stützpunkten dieses weitgespannten Archipels rasch und effizient zu übersetzen? Für Benedetto Bordone musste Venedig prädestiniert dafür sein, von Europa aus ein weltweites Beziehungsgeflecht aufzubauen, das die eigene Inselwelt in eine globale, weltweit sich erstreckende Inselwelt umzuwandeln verstünde. Eine Propagandaschrift für die im globalen Wettrennen ins Hintertreffen geratene Republik der Serenissima? Ganz zweifellos ist eine solche Deutung nicht gänzlich von der Hand zu weisen, sondern in den Entwurf des Isolario eingewoben.

So spiegeln sich im Wasser jenes gewaltigen Sees, in dessen Mitte sich die Hauptstadt des Aztekenreichs als Insel erhebt, die Umrisse von Venedig - ganz so, wie man den Stadtplan von Temistitan als unzweifelhaft venezianisiert bezeichnen könnte. In der Beschreibung oder besser Inszenierung der Schönheit dieser Stadt, die mit menschlicher Sprache ${ }^{18}$ kaum zum Ausdruck zu bringen sei, stoßen wir folglich nicht nur auf eine die gesamte Landschaft umfassende Tropikalisierung eines locus amoenus, ${ }^{19}$ sondern auch auf eine Hommage an jene Lagunenstadt, die parallel zu ihrer wirtschaftlichen und politischen Macht zu einem der Zentren für die Zirkulation von Wissen über die außereuropäische Welt geworden war. Venedig war zu einem Kreuzungspunkt von Informationen über die Ausweitung europäischer Handelsnetze und europäischer 'Entdeckungen' geworden.

18 Bordone, Libro, Bl. 9 und 12.

19 Ebda., S. 12. 
Denn jenseits aller Fehler, welche die Werke der „antichi“20 - und man fühlt hier den ganzen Stolz eines Menschen der Renaissance - hinterlassen hätten, ist ein neues Wissen über den gesamten Erdkörper und dessen Bewohner entstanden, das Benedetto Bordone in der erprobten Form eines Inselbuches zusammenzuführen versuchte, welches sich nun aber nicht mehr nur auf einen Teil des Mittelmeeres, sondern auf die gesamte Welt, auf - so der Titel stolz - tutte l'isole del mondo bezieht. Die Welt hatte sich für die europäische Globalisierung gerundet: Die erste Phase beschleunigter Globalisierung war im vollen Gange. Und ständig tauchten neue Inseln auf, die es in die Machtbeziehungen europäischer Kolonialreiche $\mathrm{zu}$ integrieren galt.

Dieses Wissen von der Welt bündelte sich in Europa; und so schien es aus dieser Perspektive auch Europa vorbehalten zu sein, die so unterschiedlichen Inseln der Welt translokal und transareal miteinander in Verbindung zu setzen. Wohlgemerkt: von Europa aus und im Interesse der Alten Welt. Allein die Europäer und in besonderem Maße die Venezianer - die gegenüber den Portugiesen und Spaniern als Handelsimperium von den Gewürzinseln wie den Luxusgütern des Fernen Ostens abgeschnitten zu werden drohten - verfügten über die infrastrukturellen Mittel und das dafür notwendige Wissen und Kapital, das es nun einzusetzen galt. Der Isolario des aus Padua nach Venedig gekommenen Künstlers und Kartographen schloss bewusst Insel und Meer mit dem Wissen zusammen und untermauerte den fortgesetzten Anspruch der Lagunenstadt auf den Rang einer Großmacht im Weltmaßstab. Sein Isolario beruhte auf Reiseberichten und bildete selbst in seinem Textteil die spezifische Form eines Reiseberichts, in dem es um keine Linearität ging.

Doch Bordones Insularium ist ohne Zweifel weitaus mehr als ein provenezianisches Plädoyer. Denn zugleich kommt in Bordones Werk anders als in den zusammenhängenden, am Kontinentalen ausgerichteten graphischen wie skripturalen Repräsentationen der Welt eine andere Weltanschauung zum Ausdruck, die auf die Diversität aller Erscheinungen und Ausdrucksformen hin angelegt ist. Betrachten wir etwa die Darstellung des Archipels der Karibik, welche in vielerlei Hinsicht noch den Informationsstand und die auf diesen Raum projizierten Mythen der Antike reflektiert, so bemerken wir rasch, wie sehr sich die einzelnen kleineren Inseln nicht nur naturräumlich und naturgeschichtlich, sondern vor allem funktional und kulturell voneinander unterscheiden.

Bei den später so genannten Großen Antillen, die eine größere Landmasse repräsentieren, findet sich wiederum eine starke Binnendifferenzierung, die ebenfalls viele Bereiche von Natur und Kultur miteinschließt. Die Inseln sind

20 Ebda., unpaginierter Auftakt, S. 3. 
hochgradig 'individualisiert', auch wenn bei Benedetto Bordone das in anderen Quellen Vorgefundene wie das Erfundene dominiert und nicht im eigenen Erlebten verankert werden kann. Gleichwohl präsentiert Bordone sein Inselbuch im Sinne dreier verschiedener Parcours, die deutlich an die Wege eines Reisenden, nicht aber an die eines Gelehrten am heimischen Schreibtisch erinnern. Denn sein Isolario ist nicht zuletzt ein Reisebuch.

Das vielleicht beste Beispiel für die Binnendifferenzierung der Großen Antillen bildet die Insel Cuba (vgl. Abb. 11: Karte der Insel Kuba, Benedetto Bordone, 1528), deren weltweit wohl erste separate Darstellung im Kartenbild des Isolario von einem im Verhältnis zu anderen Inseln umfangreichen Schrift-Text begleitet wird. Diese Insel, die Christoph Columbus am 20. Oktober 1492 nicht nur für „das Schönste, was Augen je gesehen“, ${ }^{21}$ sondern auch wegen ihrer Längenerstreckung für einen Kontinent hielt, erscheint auch bei Bordone als „sehr große Insel“ (isola molto grande). ${ }^{22}$ Sie sei wie ein Krokodil geformt und schare mehr als siebenhundert weitere Inseln und Inselchen um sich, die allesamt bewohnt seien. ${ }^{23}$ Cuba selbst erscheint damit - ganz so, wie es bis heute in kubanischen Schulbüchern steht - als Archipel für sich und nicht als eine einfache Insel. Die Hauptinsel ist gesäumt von kleinen Inseln, cayos und winzigen Inselgruppen, die vor der Küste liegen - ganz so, wie es Guillermo Cabrera Infante in seinem Vista del amanecer en el trópico geradezu mythologisch entwerfen sollte.

Benedetto Bordone, der offenkundig nur aus schriftlichen, nicht aber aus kartographischen Quellen von der (angeblichen und bis heute tradierten) Krokodilsform der Insel erfahren haben dürfte und sich redlich bemühte, nach eigener Phantasie eine derartige Form im Kartenbild entstehen zu lassen, betonte die „hohe Diversität bei den Sprachen und Sitten der Menschen“, ${ }^{24}$ durch die sich die große Insel Cuba auszeichne. Dies gelte nicht nur für die auf der Insel benutzten Sprach- und Lebensformen, sondern auch für die naturräumliche Ausstattung, für Fauna und Flora der Insel, in deren Binnenland sich dichte Urwälder ausdehnten und ausreichend Holz für den dringend benötigten Schiffbau der Europäer bereitstellten.

Bordones Isolario lässt eine Welt aus Inseln entstehen, in der sich große und kleine Landflächen, Eilande und Kontinente wie Inseln zueinander verhalten und von einer jeweils sehr stark ausgeprägten Eigen-Logik charakterisiert sind.

21 Colón, Cristóbal: Los cuatro viajes. Testamento. Edición de Consuelo Varela. Madrid: Alianza Editorial 1986, S. 82.

22 Bordone, Libro, Bl. XIII.

23 Ebda.

24 Ebda., Bl. XIIII: „molta diversita di parlare, di costumi di huomini“. 
Wir haben es in einem umfassenden Sinne mit einer wirklichen Landschaft der Theorie zu tun: einer Landschaft, welche die in ihr enthaltene Theorie visualisiert und vor Augen führt. Die Vielzahl an Eigen-Logiken führt zu einer Viel-Logik, zu einer Polylogik, die mit der Polyperspektivität einhergeht. Die von Bordone ersonnene Insel Cuba führt vor, um welches Modell, um welches Weltverständnis es dem in Venedig lebenden Maler zu tun war.

Damit erscheint die Welt in ihrer Gesamtheit nicht nur als eine Insel, wie sie in einer späten Blüte des Genres im Jahre 1697 im Isolario von Vincenzo Maria Coronelli - „ein Weltatlas in Form eines Inselbuchs““25 - konfiguriert wurde, sondern als eine Welt, die aus Inseln gebildet ist, die ihrerseits (wie Cuba) aus Inseln bestehen oder deren Machtzentren (wie Venedig) aus Inseln modelliert sind. Die ganze Welt ist in Bordones Isolario im fraktalen Sinne eine Insel aus Inseln aus Inseln - eine Sichtweise, die nicht nur die Vektorisierung, sondern auch die Venezianisierung der künstlerisch-kartographischen Projektionen unserer Erdkugel mit sich bringt. Das Insel-Fraktal wird gleichsam zur graphischen Welt-Formel: eine Landschaft der Theorie wird geboren.

Folglich kann eine Insel auch stets eine andere Insel bergen (oder verbergen) sowie relational auf andere Inseln verweisen. Nicht umsonst hatte Christoph Columbus als aufmerksamer Leser von Marco Polo die Insel Cuba mit jener Insel Cipango oder Cipangu identifiziert, von deren Reichtum in Il Milione so ausführlich die Rede war. So hieß es zu Beginn dieser Beschreibung Cipangos in Marco Polos ursprünglich in den Jahren 1298 und 1299 erstellten Bericht:

\footnotetext{
Gehen wir nun zur Beschreibung der Regionen Indiens über; dabei beginnen wir mit der Insel Ciampagu, die eine Insel im Osten ist, draußen im offenen Meer, tausendvierhundert Meilen von der Küste von Mangi entfernt. Sie ist äußerst groß, und ihre Bewohner, die weiß sind und ein hübsches Aussehen besitzen, sind Götzendiener und haben einen König, wobei sie an niemanden anderen Tribut zahlen müssen. Hier gibt es Gold in übergroßer Fülle (abundancia), doch der Monarch erlaubt nicht leicht, daß es von der Insel ausgeführt werde, so daß nur wenige Händler dorthin fahren und selten Schiffe anderer Regionen in ihre Häfen gelangen. Der König der Insel besitzt einen großen Palast mit Dächern aus sehr feinem Gold, so wie bei uns die Kirchen Bleidächer tragen. Die Fenster dieses Palastes sind allesamt reich mit Gold verziert, und die Fußböden der Säle und vieler Wohnräume sind mit goldenen Dielen versehen, welche zwei Finger dick sind. Hier gibt es Perlen in äußerster Fülle, rund und dick und von roter Farbe, die an Preis und Wert die weißen Samenperlen übertreffen. Es gibt auch viele Edelsteine, so daß die Insel Ciampagu auf wunderbare Weise reich ist. ${ }^{26}$
}

25 Karrow, Benedetto Bordone, S. 93: „a world atlas in the form of an island book.“

26 Gil, Juan (Hg.): El libro de Marco Polo anotado por Cristóbal Colón. El libro de Marco Polo versión de rodrigo de Santaella. Edición, introducción y notas de Juan Gil. Madrid: Alianza Editorial 1987, S. 132. Übersetzungen von Texten, die nach dem fremdsprachigen Original zitiert werden, stammen - wo nicht anders angegeben - vom Verfasser (O.E.). Damit sollen die 


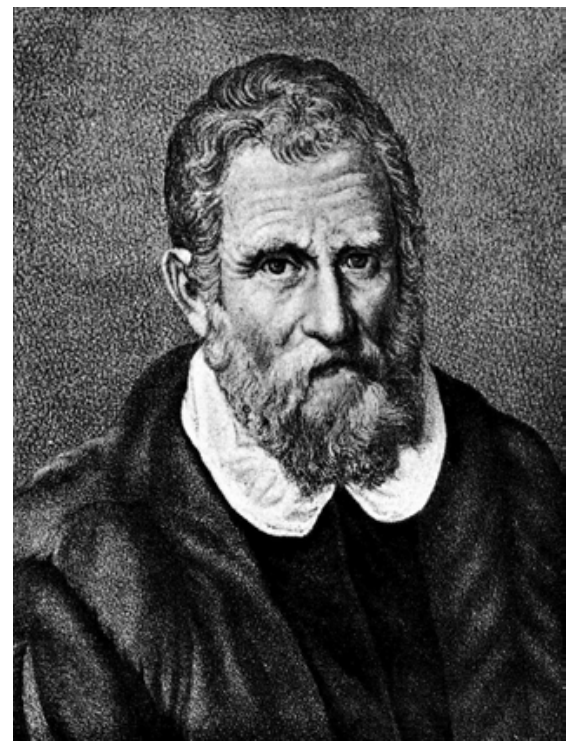

Abb. 14: Marco Polo (Venedig?, um 1254 - ebenda, 1324).

Dieses Zitat aus einer von keinem Geringeren als Christoph Columbus kommentierten Ausgabe von Marco Polo lässt keinerlei Zweifel aufkommen: Spätestens mit Marco Polos Il Milione begann sich jenes koloniale Kaleidoskop zu drehen, in dessen Bewegungen sich Finden, Erfinden und Erleben, mithin 'Fakten', 'Fiktionen' und Leben, zugleich aber Inselwelten Asiens und Inselwelten der Amerikas auf immer wieder neue Weise miteinander transareal kombinierten und austauschten. Reisen und über Reisen Schreiben bedürfen daher einer weiteren Komponente, um sich zu einem Dreiklang zu runden: Sie bedürfen des Lesens, der Lektüre - und zwar möglichst der Lektüre anderer Reiseberichte. So ließe sich mit Blick auf Christoph Columbus mit guten Gründen sagen: Die erste Reise eines Europäers durch die Karibik war eine Reise des Lesens. Und Le livre de Marco Polo citoyen de Venise, dit Milione, où l'on conte les merveilles du monde hatte daran einen wohl kaum zu überschätzenden Anteil. Auf eben diese Weise gelangte von Beginn an Asien in die Karibik, nach Amerika.

fremdsprachigen Passagen für die deutschsprachige Leserschaft leichter zugänglich gemacht werden, wobei zugleich eine möglichst große Nähe zum Original sichergestellt wird. Auf vorhandene (und bisweilen ausgezeichnete) Übersetzungen, die ihren je eigenen Deutungsmustern folgen, wurde daher nur ausnahmsweise zurückgegriffen. 